\title{
Electronic Supplementary Materials: Rising Rates And Widening Socio-economic Disparities In Diabetic Ketoacidosis In Type 1 Diabetes In Scotland: A Nationwide Retrospective Cohort Observational Study
}

\author{
Joseph E. O'Reilly ${ }^{1}$, Anita Jeyam¹ ${ }^{1}$,Thomas M. Caparrotta ${ }^{1}$, Joseph Mellor ${ }^{2}$, \\ Andreas Hohn ${ }^{1}$, Paul M. McKeigue ${ }^{2}$, Stuart J. McGurnaghan ${ }^{1}$, Luke A.K. Blackbourn ${ }^{1}$, \\ Rory McCrimmon ${ }^{3}$, Sarah Wild ${ }^{2}$, John Petrie ${ }^{4}$, John A. McKnight ${ }^{5}$, Brian Kennon ${ }^{6}$, \\ John Chalmers ${ }^{7}$, Sam Phillip ${ }^{8}$, Graham Leese ${ }^{9}$, \\ Robert S. Lindsay ${ }^{4}$, Naveed Sattar ${ }^{4}$, Fraser Gibb ${ }^{10}$, and Helen M. Colhoun ${ }^{1,11}$, \\ for the Scottish Diabetes Research Network Epidemiology Group
}

${ }^{1}$ MRC Institute of Genetic and Molecular Medicine, University of Edinburgh, UK

${ }^{2}$ Usher Institute, University of Edinburgh, UK

${ }^{3}$ Division of Molecular and Clinical medicine, University of Dundee, Dundee, UK

${ }^{4}$ Institute of Cardiovascular and Medical Sciences, University of Glasgow, Glasgow, UK

${ }^{5}$ Western General Hospital, NHS Lothian, Edinburgh, UK

${ }^{6}$ Queen Elizabeth University Hospital, Glasgow, UK

${ }^{7}$ Diabetes Centre, Victoria Hospital, Kirkcaldy, UK

${ }^{8}$ Grampian Diabetes Research Unit, Diabetes Centre, Aberdeen Royal Infirmary, Aberdeen, UK

${ }^{9}$ Ninewells Hospital, NHS Tayside, Dundee, UK

${ }^{10}$ Royal Infirmary of Edinburgh, NHS Lothian, Edinburgh, UK

${ }^{11}$ Department of Public Health, NHS Fife, Kirkcaldy, UK

\section{ESM Statistical Techniques}

As DKA event counts are a non-negative integer valued outcome, we applied a Poisson likelihood function in the GLM framework with an offset equal to the log of the duration of observable person-time. To account for potential over dispersion of events caused by repeated measurement of the outcome we applied a hierarchical regression framework with individual subject specific random intercepts. All regression analyses were performed with the $\mathrm{R}$ package brms (1).

As crude DKA event rates varied non-linearly with age, when DKA event counts were the outcome variable we constructed the age covariate as age-bands consisting of ten year increments. When DKA case-fatality was the outcome we included a single linear term for age instead. Despite the total quantity of missing data being relatively small, a number of covariates had a 'not completely at random' distribution of missing data. This was particularly driven by missing data for biochemical tests in younger individuals. Accordingly, we employed multiple imputation, as implemented in the R package Amelia (2). Regression analyses were performed on each one of 5 imputed datasets, and posterior samples were combined across replicate analyses. 
For Bayesian regression analyses a diffuse improper prior was assigned to all regression coefficients. Samples from the posterior distribution were obtained using Stan via the brms R package and were summarised as $95 \%$ credible intervals (CrI) and median posterior estimates. For each imputed dataset we used two independent chains of 2000 generations which were subsequently combined when assessing convergence. Convergence was accepted when all covariates possessed an r-hat close to 1.00 and no divergent transitions were reported. Individual chains were also visually inspected for stationarity. We calculated an equivalent to the classical p-value for each regression coefficient, assessed at an approximation of the maximum likelihood estimate. This was achieved by fitting a quadratic function to the sampled likelihood and obtaining an approximation of the maximum-likelihood estimate and standard error from this function.

\section{ESM Figures}

ESM Figure 1. Distribution of risk factors over the study period, by SIMD quintile

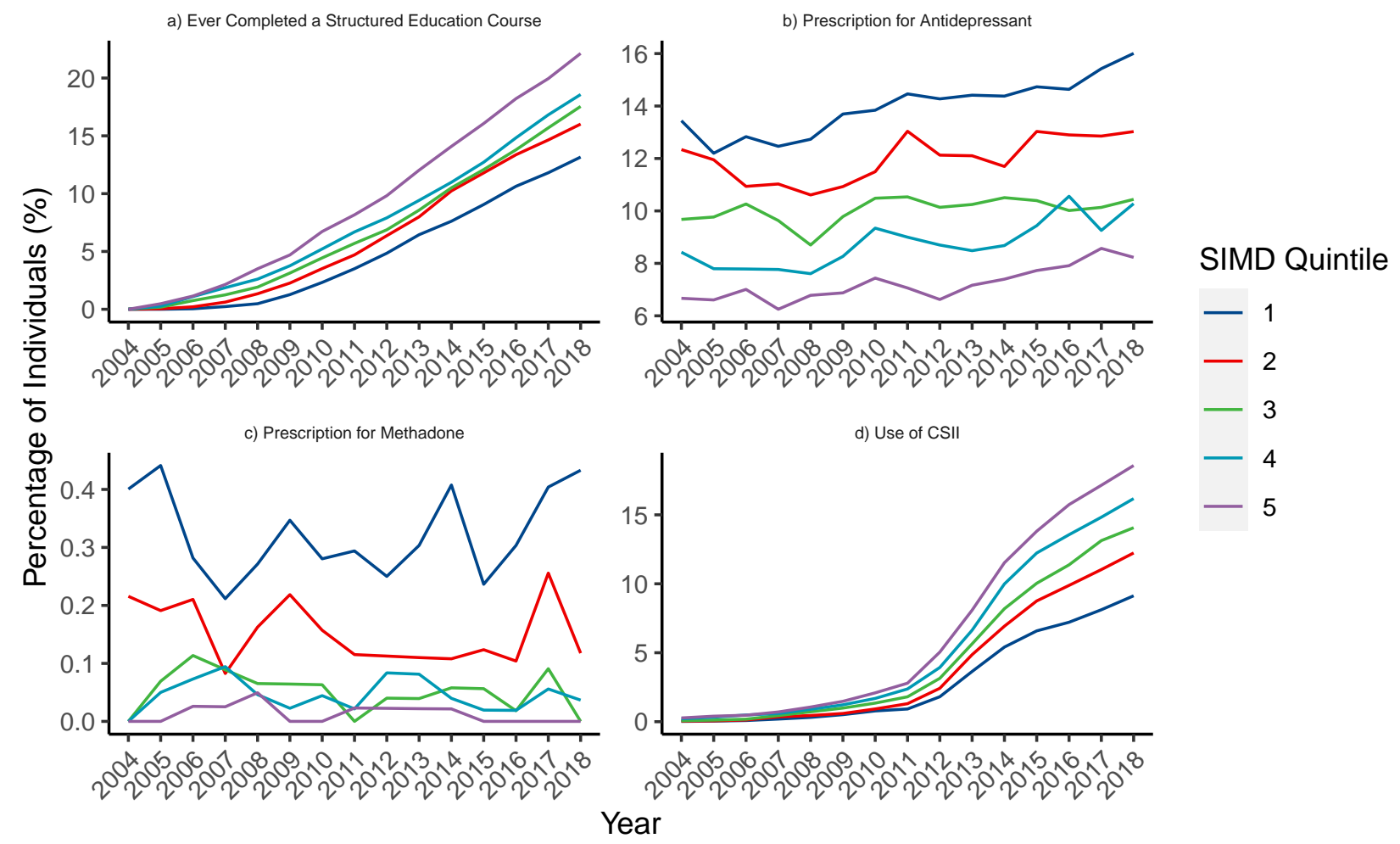




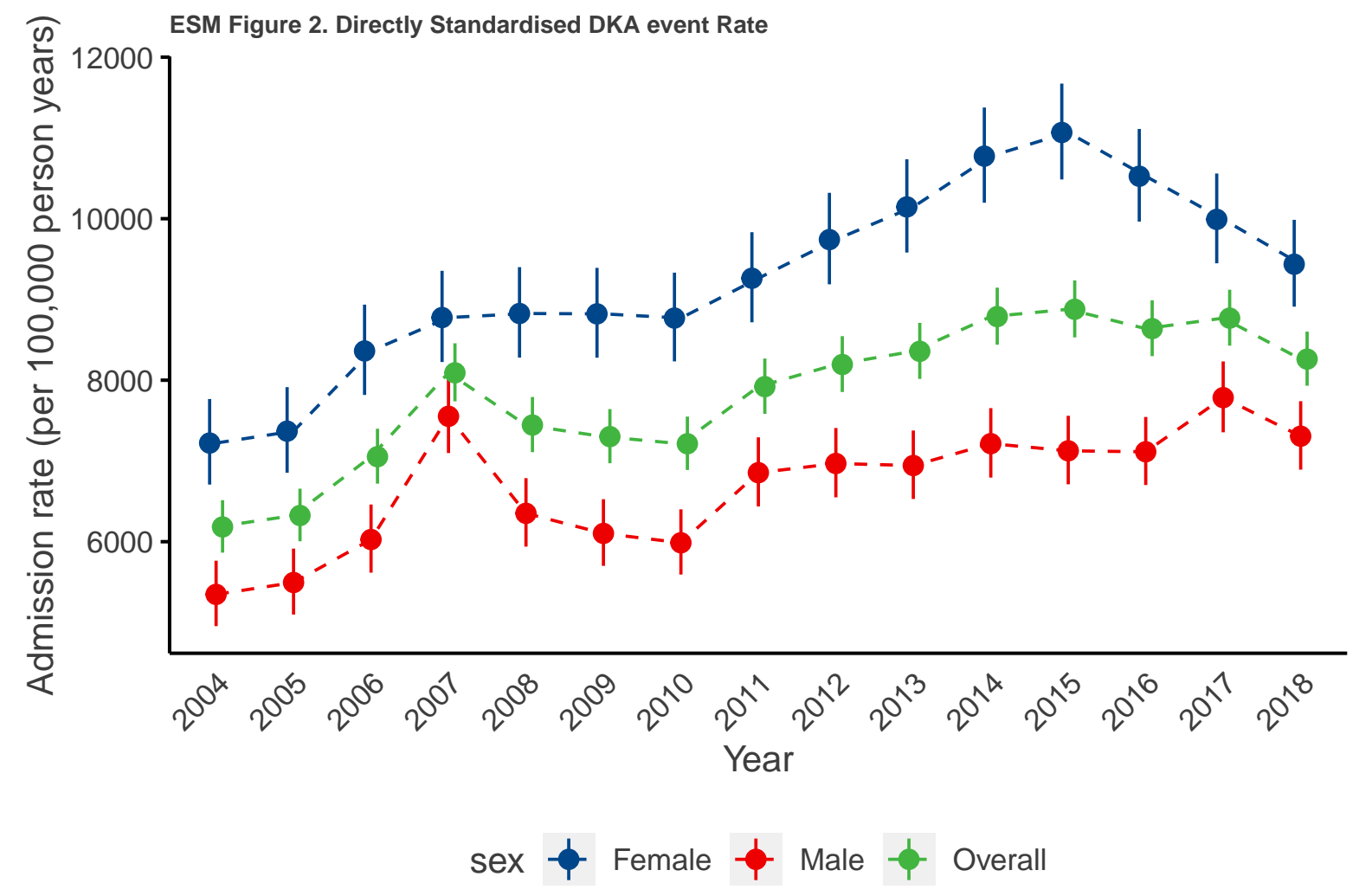

ESM Figure 3. Percentage of diabetes diagnoses presenting with DKA

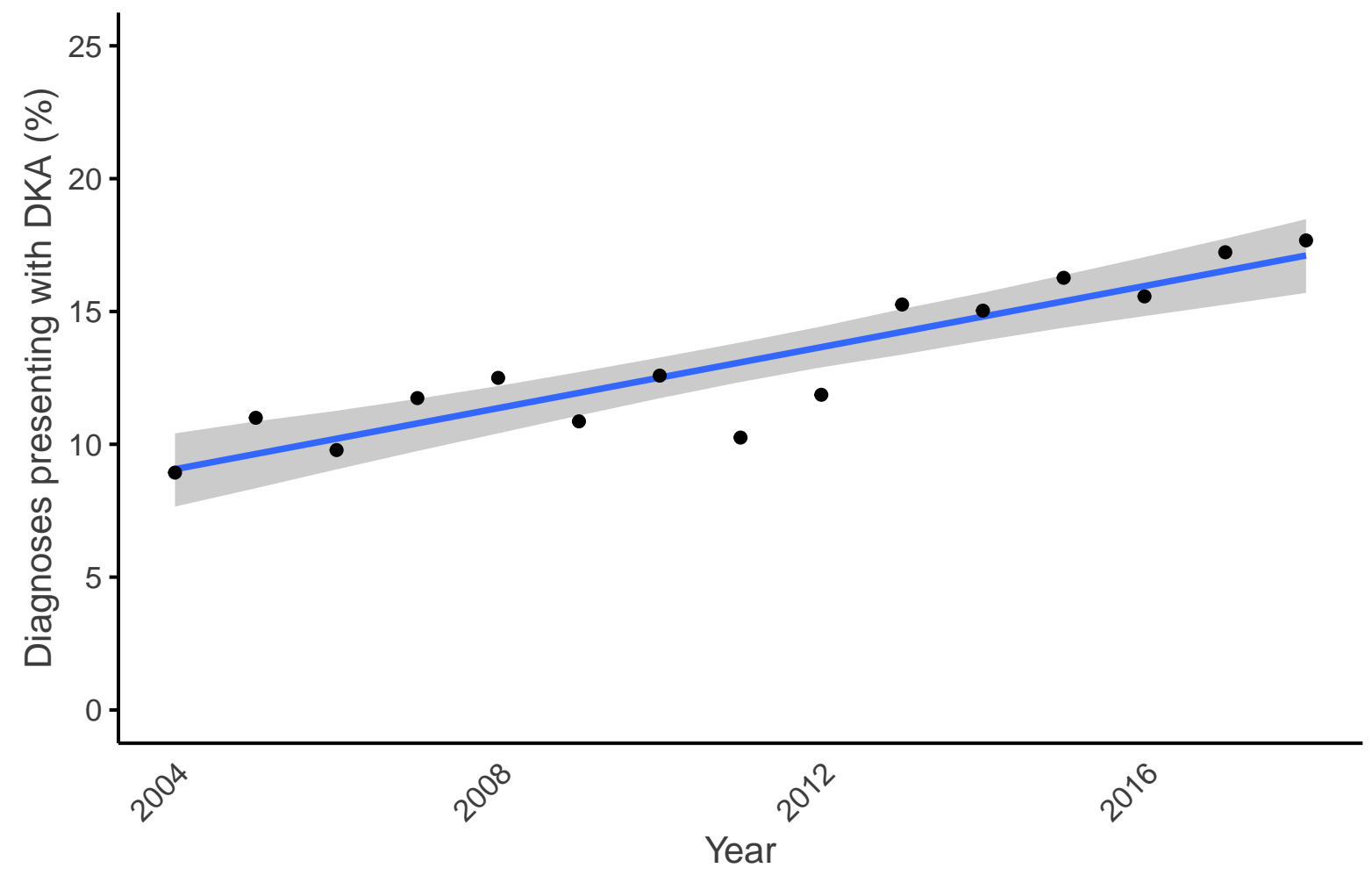




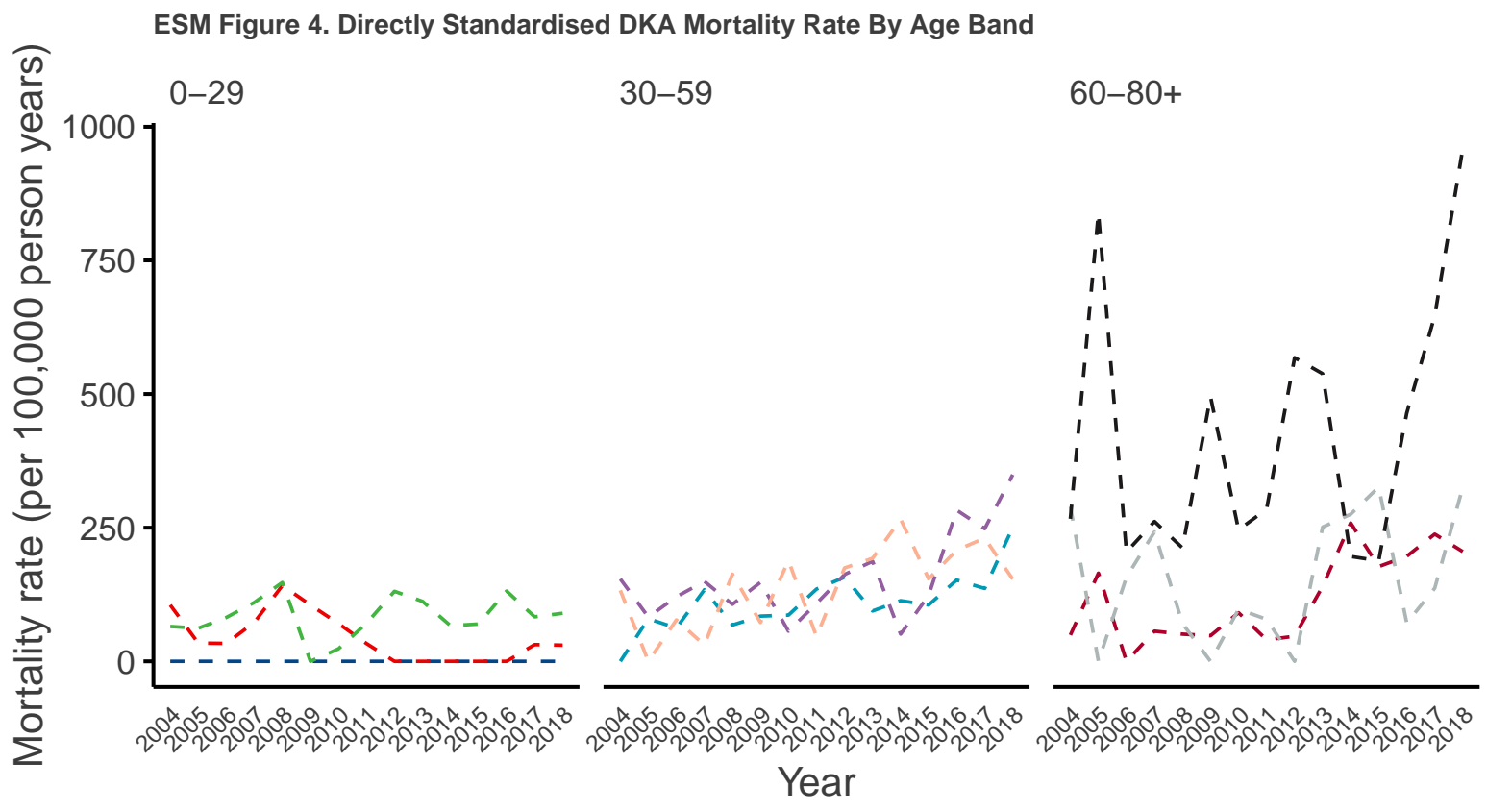

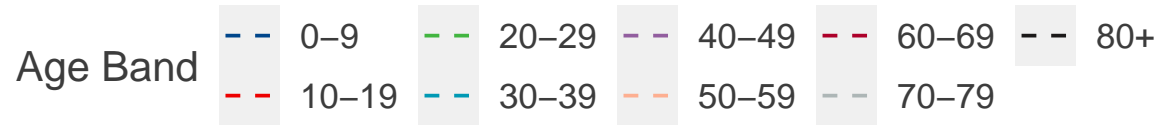

ESM table 1. Cohort Characteristics At Time Of Entry To Study. SD = Standard Deviation

\begin{tabular}{|c|c|c|c|c|c|c|c|c|}
\hline & No DKA & $(\mathrm{SD})$ & $1 \mathrm{DKA}$ & $(\mathrm{SD})$ & $2 \mathrm{DKA}$ & $(\mathrm{SD})$ & $>2 \mathrm{DKA}$ & $(\mathrm{SD})$ \\
\hline $\mathrm{N}$ & 27523.00 & - & 5722.00 & - & 1750.00 & - & 2916.00 & - \\
\hline Mean age at cohort entry & 37.35 & $(19.16)$ & 29.56 & $(18.77)$ & 28.89 & $(18.55)$ & 26.37 & $(16.14)$ \\
\hline Mean age at diagnosis of type 1 diabetes & 24.72 & $(16.98)$ & 21.11 & $(15.64)$ & 19.51 & $(14.36)$ & 18.01 & $(12.91)$ \\
\hline Male $(\%)$ & 57.22 & - & 54.32 & - & 51.20 & - & 47.94 & - \\
\hline Mean diabetes duration at cohort entry & 12.66 & $(12.75)$ & 8.50 & $(11.38)$ & 9.41 & $(11.50)$ & 8.39 & $(10.15)$ \\
\hline Mean $\mathrm{HbA1c}(\mathrm{mmol} / \mathrm{mol})$ & 72.23 & $(19.30)$ & 77.28 & $(21.46)$ & 80.67 & $(21.25)$ & 84.49 & $(22.79)$ \\
\hline Prescription for methadone $(\%)$ & 0.07 & - & 0.16 & - & 0.46 & - & 0.55 & - \\
\hline Prescription for any antidepressant medication (\%) & 8.66 & - & 8.74 & - & 10.46 & - & 12.14 & - \\
\hline Percentage of individuals in SIMD $1(\%)$ & 16.80 & - & 22.67 & - & 28.97 & - & 35.15 & - \\
\hline Percentage of individuals in SIMD $2(\%)$ & 19.14 & - & 21.71 & - & 22.46 & - & 22.36 & - \\
\hline Percentage of individuals in SIMD 3 (\%) & 19.33 & - & 17.21 & - & 15.14 & - & 14.88 & - \\
\hline Percentage of individuals in SIMD $4(\%)$ & 18.46 & - & 16.59 & - & 14.06 & - & 9.50 & - \\
\hline Percentage of individuals in SIMD $5(\%)$ & 17.84 & - & 14.37 & - & 10.06 & - & 6.62 & - \\
\hline
\end{tabular}


ESM table 2. Age-Band Stratified DKA Event IRR Estimates Obtained With Multivariable Poisson Regression

\begin{tabular}{|c|c|c|c|c|c|}
\hline Covariate & IRR & Q2.5 & Q97.5 & Approx. p-value & Age Band \\
\hline Year & 1.044 & 1.039 & 1.050 & $<0.001$ & $0-29$ \\
\hline Age $1-10$ & Ref. & - & - & - & $0-29$ \\
\hline Age 11-19 & 1.087 & 0.994 & 1.189 & 0.049 & $0-29$ \\
\hline Age $20-29$ & 0.817 & 0.738 & 0.905 & $<0.001$ & $0-29$ \\
\hline Median HbA1c $<7.5 \%,<58(\mathrm{mmol} / \mathrm{mol})$ & Ref. & - & - & - & $0-29$ \\
\hline Median HbA1c 7.5-9.0 \%, 58-75 (mmol/mol) & 1.628 & 1.476 & 1.797 & $<0.001$ & $0-29$ \\
\hline Median HbA1c 9.1-10.0 \%, 76-86 $(\mathrm{mmol} / \mathrm{mol})$ & 3.227 & 2.929 & 3.570 & $<0.001$ & $0-29$ \\
\hline Median HbA1c $>10.0 \%,>86(\mathrm{mmol} / \mathrm{mol})$ & 6.458 & 5.867 & 7.103 & $<0.001$ & $0-29$ \\
\hline Diabetes duration & 0.966 & 0.961 & 0.971 & $<0.001$ & $0-29$ \\
\hline Male & 0.766 & 0.720 & 0.815 & $<0.001$ & $0-29$ \\
\hline Female & Ref. & - & - & - & $0-29$ \\
\hline SIMD Quintile 1 (most deprived) & Ref. & - & - & - & $0-29$ \\
\hline SIMD Quintile 2 & 0.732 & 0.661 & 0.830 & $<0.001$ & $0-29$ \\
\hline SIMD Quintile 3 & 0.622 & 0.564 & 0.694 & $<0.001$ & $0-29$ \\
\hline SIMD Quintile 4 & 0.506 & 0.454 & 0.558 & $<0.001$ & $0-29$ \\
\hline SIMD Quintile 5 (least deprived) & 0.463 & 0.413 & 0.519 & $<0.001$ & $0-29$ \\
\hline Current CSII therapy & 0.741 & 0.672 & 0.817 & $<0.001$ & $0-29$ \\
\hline Current methadone prescription & 1.586 & 1.154 & 2.145 & 0.009 & $0-29$ \\
\hline Current antidepressant prescription & 1.502 & 1.436 & 1.573 & $<0.001$ & $0-29$ \\
\hline Completed a structured education course & 0.968 & 0.884 & 1.059 & 0.490 & $0-29$ \\
\hline Year & 1.066 & 1.060 & 1.072 & $<0.001$ & $30-59$ \\
\hline Age $30-39$ & Ref. & - & - & - & $30-59$ \\
\hline Age $40-49$ & 0.890 & 0.839 & 0.945 & $<0.001$ & $30-59$ \\
\hline Age $50-59$ & 0.896 & 0.828 & 0.972 & 0.006 & $30-59$ \\
\hline Median HbA1c $<7.5 \%,<58(\mathrm{mmol} / \mathrm{mol})$ & Ref. & - & - & - & $30-59$ \\
\hline Median HbA1c $7.5-9.0 \%, 58-75(\mathrm{mmol} / \mathrm{mol})$ & 1.512 & 1.370 & 1.676 & $<0.001$ & $30-59$ \\
\hline Median HbA1c $9.1-10.0 \%, 76-86(\mathrm{mmol} / \mathrm{mol})$ & 2.539 & 2.295 & 2.811 & $<0.001$ & $30-59$ \\
\hline Median HbA1c $>10.0 \%,>86(\mathrm{mmol} / \mathrm{mol})$ & 3.673 & 3.302 & 4.088 & $<0.001$ & $30-59$ \\
\hline Diabetes duration & 0.974 & 0.970 & 0.977 & $<0.001$ & $30-59$ \\
\hline Male & 0.821 & 0.760 & 0.887 & $<0.001$ & $30-59$ \\
\hline Female & Ref. & - & - & - & $30-59$ \\
\hline SIMD Quintile 1 (most deprived) & Ref. & - & - & - & $30-59$ \\
\hline SIMD Quintile 2 & 0.728 & 0.661 & 0.803 & $<0.001$ & $30-59$ \\
\hline SIMD Quintile 3 & 0.539 & 0.482 & 0.612 & $<0.001$ & $30-59$ \\
\hline SIMD Quintile 4 & 0.453 & 0.392 & 0.513 & $<0.001$ & $30-59$ \\
\hline SIMD Quintile 5 (least deprived) & 0.359 & 0.312 & 0.415 & $<0.001$ & $30-59$ \\
\hline Current CSII therapy & 1.072 & 0.915 & 1.248 & 0.405 & $30-59$ \\
\hline Current methadone prescription & 1.437 & 1.162 & 1.770 & 0.002 & $30-59$ \\
\hline Current antidepressant prescription & 1.497 & 1.424 & 1.572 & $<0.001$ & $30-59$ \\
\hline Completed a structured education course & 0.712 & 0.636 & 0.794 & $<0.001$ & $30-59$ \\
\hline Year & 1.096 & 1.081 & 1.110 & $<0.001$ & $60+$ \\
\hline Age $60-69$ & Ref. & - & - & - & $60+$ \\
\hline Age $70-79$ & 1.482 & 1.317 & 1.678 & $<0.001$ & $60+$ \\
\hline Age $80+$ & 2.417 & 2.017 & 2.893 & $<0.001$ & $60+$ \\
\hline Median HbA1c $<7.5 \%,<58(\mathrm{mmol} / \mathrm{mol})$ & Ref. & - & - & - & $60+$ \\
\hline Median HbA1c 7.5-9.0 \%, 58-75 (mmol/mol) & 1.601 & 1.369 & 1.884 & $<0.001$ & $60+$ \\
\hline Median HbA1c 9.1-10.0 \%, 76-86 $(\mathrm{mmol} / \mathrm{mol})$ & 2.737 & 2.295 & 3.257 & $<0.001$ & $60+$ \\
\hline Median HbA1c $>10.0 \%,>86(\mathrm{mmol} / \mathrm{mol})$ & 4.493 & 3.765 & 5.394 & $<0.001$ & $60+$ \\
\hline Diabetes duration & 1.008 & 1.003 & 1.013 & 0.001 & $60+$ \\
\hline Male & 0.824 & 0.710 & 0.954 & 0.014 & $60+$ \\
\hline Female & Ref. & - & - & - & $60+$ \\
\hline SIMD Quintile 1 (most deprived) & Ref. & - & - & - & $60+$ \\
\hline SIMD Quintile 2 & 0.943 & 0.715 & 1.200 & 0.497 & $60+$ \\
\hline SIMD Quintile 3 & 0.662 & 0.539 & 0.811 & $<0.001$ & $60+$ \\
\hline SIMD Quintile 4 & 0.726 & 0.580 & 0.903 & 0.002 & $60+$ \\
\hline SIMD Quintile 5 (least deprived) & 0.718 & 0.569 & 0.907 & 0.004 & $60+$ \\
\hline Current CSII therapy & 1.574 & 1.026 & 2.412 & 0.043 & $60+$ \\
\hline Current methadone prescription & 3.572 & 0.097 & 56.836 & 0.807 & $60+$ \\
\hline Current antidepressant prescription & 1.832 & 1.631 & 2.052 & $<0.001$ & $60+$ \\
\hline Completed a structured education course & 0.571 & 0.418 & 0.778 & $<0.001$ & $60+$ \\
\hline
\end{tabular}


ESM table 3. Recurrent DKA IRR Estimates Obtained With Multivariable Logistic Regression

\begin{tabular}{lllll}
\hline Covariate & IRR & Q2.5 & Q97.5 & Approx. p-value \\
\hline Year & 1.037 & 1.030 & 1.044 & $<0.001$ \\
Age 1-10 & Ref. & - & - & - \\
Age 11-19 & 3.924 & 2.840 & 5.569 & $<0.001$ \\
Age 20-29 & 2.696 & 1.954 & 3.829 & $<0.001$ \\
Age 30-39 & 1.585 & 1.135 & 2.259 & 0.004 \\
Age 40-49 & 0.987 & 0.705 & 1.419 & 0.933 \\
Age 50-59 & 1.054 & 0.755 & 1.502 & 0.664 \\
Age 60-69 & 0.982 & 0.684 & 1.433 & 0.963 \\
Age 70-79 & 1.546 & 1.063 & 2.290 & 0.017 \\
Age 80+ & 2.124 & 1.395 & 3.303 & 0.001 \\
Median HbA1c $<7.5 \%,<58(\mathrm{mmol} / \mathrm{mol})$ & Ref. & - & - & - \\
Median HbA1c 7.5-9.0\%, 58-75 (mmol/mol) & 2.268 & 1.872 & 2.766 & $<0.001$ \\
Median HbA1c 9.1-10.0\%, 76-86 (mmol/mol) & 5.821 & 4.860 & 7.055 & $<0.001$ \\
Median HbA1c $>10.0 \%,>86(\mathrm{mmol} / \mathrm{mol})$ & 16.361 & 13.610 & 19.734 & $<0.001$ \\
Diabetes duration & 0.994 & 0.991 & 0.997 & $<0.001$ \\
Male & 0.742 & 0.700 & 0.785 & $<0.001$ \\
Female & Ref. & - & - & - \\
SIMD Quintile 1 (most deprived) & Ref. & - & - & - \\
SIMD Quintile 2 & 0.686 & 0.634 & 0.743 & $<0.001$ \\
SIMD Quintile 3 & 0.556 & 0.511 & 0.604 & $<0.001$ \\
SIMD Quintile 4 & 0.398 & 0.360 & 0.440 & $<0.001$ \\
SIMD Quintile 5 (least deprived) & 0.323 & 0.283 & 0.368 & $<0.001$ \\
Current CSII therapy & 0.796 & 0.660 & 0.954 & 0.017 \\
Current methadone prescription & 3.346 & 2.472 & 4.444 & $<0.001$ \\
Current antidepressant prescription & 2.250 & 2.103 & 2.406 & $<0.001$ \\
Completed a structured education course & 0.735 & 0.640 & 0.838 & $<0.001$ \\
\hline
\end{tabular}


ESM table 4. Case-Fatality Odds-Ratio Estimates Obtained With Multivariable Logistic Regression

\begin{tabular}{lllll}
\hline Covariate & OR & Q2.5 & Q97.5 & Approx. p-value \\
\hline Year & 1.026 & 1.003 & 1.050 & 0.018 \\
Age & 1.045 & 1.038 & 1.051 & $<0.001$ \\
Median HbA1c $<7.5 \%,<58(\mathrm{mmol} / \mathrm{mol})$ & Ref. & - & - & - \\
Median HbA1c $7.5-9.0 \%, 58-75(\mathrm{mmol} / \mathrm{mol})$ & 0.609 & 0.417 & 0.906 & 0.012 \\
Median HbA1c $9.1-10.0 \%, 76-86(\mathrm{mmol} / \mathrm{mol})$ & 0.657 & 0.445 & 0.997 & 0.047 \\
Median HbA1c $>10.0 \%,>86(\mathrm{mmol} / \mathrm{mol})$ & 0.717 & 0.498 & 1.081 & 0.106 \\
Diabetes duration & 1.009 & 1.002 & 1.017 & 0.016 \\
Male & 2.322 & 1.915 & 2.816 & $<0.001$ \\
Female & Ref. & - & - & - \\
SIMD Quintile 1 (most deprived) & Ref. & - & - & - \\
SIMD Quintile 2 & 0.851 & 0.668 & 1.091 & 0.226 \\
SIMD Quintile 3 & 0.606 & 0.438 & 0.830 & 0.001 \\
SIMD Quintile 4 & 0.906 & 0.672 & 1.215 & 0.471 \\
SIMD Quintile 5 (least deprived) & 0.677 & 0.465 & 0.971 & 0.031 \\
Current CSII therapy & 0.533 & 0.204 & 1.179 & 0.095 \\
Current methadone prescription & 5.676 & 3.359 & 9.241 & $<0.001$ \\
Current antidepressant prescription & 1.996 & 1.640 & 2.429 & $<0.001$ \\
Completed a structured education course & 0.765 & 0.442 & 1.264 & 0.226 \\
\hline
\end{tabular}


ESM table 5. Age-Band Stratified Case-Fatality Odds-Ratio Estimates Obtained With Multivariable Logistic Regression

\begin{tabular}{|c|c|c|c|c|c|}
\hline Covariate & OR & Q2.5 & Q97.5 & Approx. p-value & Age Band \\
\hline Year & 0.966 & 0.912 & 1.024 & 0.255 & $0-29$ \\
\hline Age & 1.112 & 1.051 & 1.175 & $<0.001$ & $0-29$ \\
\hline Median HbA1c $<7.5 \%,<58(\mathrm{mmol} / \mathrm{mol})$ & 0.544 & 0.024 & 4.794 & 0.979 & $0-29$ \\
\hline Median HbA1c 7.5-9.0 \%, 58-75 (mmol $/ \mathrm{mol})$ & 0.790 & 0.308 & 1.694 & 0.335 & $0-29$ \\
\hline Median HbA1c 9.1-10.0 \%, 76-86 (mmol/mol) & 0.771 & 0.337 & 1.573 & 0.311 & $0-29$ \\
\hline Median HbA1c $>10.0 \%,>86(\mathrm{mmol} / \mathrm{mol})$ & Ref. & - & - & - & $0-29$ \\
\hline Diabetes duration & 1.028 & 0.990 & 1.069 & 0.157 & $0-29$ \\
\hline Male & 1.941 & 1.187 & 3.231 & 0.006 & $0-29$ \\
\hline Female & Ref. & - & - & - & $0-29$ \\
\hline SIMD Quintile 1 (most deprived) & Ref. & - & - & - & $0-29$ \\
\hline SIMD Quintile 2 & 1.077 & 0.586 & 1.953 & 0.781 & $0-29$ \\
\hline SIMD Quintile 3 & 0.729 & 0.321 & 1.513 & 0.316 & $0-29$ \\
\hline SIMD Quintile 4 & 0.851 & 0.333 & 1.910 & 0.480 & $0-29$ \\
\hline SIMD Quintile 5 (least deprived) & 0.598 & 0.139 & 1.816 & 0.199 & $0-29$ \\
\hline Current methadone prescription & 6.959 & 1.977 & 20.142 & 0.005 & $0-29$ \\
\hline Current antidepressant prescription & 0.813 & 0.447 & 1.420 & 0.430 & $0-29$ \\
\hline Completed a structured education course & 0.159 & 0.007 & 1.040 & 0.071 & $0-29$ \\
\hline Year & 1.045 & 1.015 & 1.076 & 0.002 & $30-59$ \\
\hline Age & 1.045 & 1.029 & 1.061 & $<0.001$ & $30-59$ \\
\hline Median HbA1c $<7.5 \%,<58(\mathrm{mmol} / \mathrm{mol})$ & 1.223 & 0.650 & 2.191 & 0.632 & $30-59$ \\
\hline Median HbA1c 7.5-9.0 \%, 58-75 (mmol/mol) & 0.766 & 0.534 & 1.076 & 0.102 & $30-59$ \\
\hline Median HbA1c 9.1-10.0 \%, 76-86 (mmol $/ \mathrm{mol})$ & 0.862 & 0.582 & 1.222 & 0.298 & $30-59$ \\
\hline Median HbA1c $>10.0 \%,>86(\mathrm{mmol} / \mathrm{mol})$ & Ref. & - & - & - & $30-59$ \\
\hline Diabetes duration & 1.011 & 1.000 & 1.022 & 0.059 & $30-59$ \\
\hline Male & 3.125 & 2.376 & 4.119 & $<0.001$ & $30-59$ \\
\hline Female & Ref. & - & - & - & $30-59$ \\
\hline SIMD Quintile 1 (most deprived) & Ref. & - & - & - & $30-59$ \\
\hline SIMD Quintile 2 & 0.775 & 0.569 & 1.043 & 0.124 & $30-59$ \\
\hline SIMD Quintile 3 & 0.625 & 0.407 & 0.916 & 0.011 & $30-59$ \\
\hline SIMD Quintile 4 & 0.930 & 0.635 & 1.334 & 0.589 & $30-59$ \\
\hline SIMD Quintile 5 (least deprived) & 0.616 & 0.344 & 1.054 & 0.047 & $30-59$ \\
\hline Current CSII therapy & 0.547 & 0.158 & 1.460 & 0.130 & $30-59$ \\
\hline Current methadone prescription & 3.987 & 2.174 & 6.986 & $<0.001$ & $30-59$ \\
\hline Current antidepressant prescription & 1.966 & 1.552 & 2.491 & $<0.001$ & $30-59$ \\
\hline Completed a structured education course & 0.828 & 0.436 & 1.484 & 0.363 & $30-59$ \\
\hline Year & 1.034 & 0.984 & 1.088 & 0.159 & $60+$ \\
\hline Age & 1.037 & 1.009 & 1.065 & 0.009 & $60+$ \\
\hline Median HbA1c $<7.5 \%,<58(\mathrm{mmol} / \mathrm{mol})$ & 2.087 & 0.927 & 4.313 & 0.076 & $60+$ \\
\hline Median HbA1c 7.5-9.0 \%, 58-75 (mmol $/ \mathrm{mol})$ & 1.098 & 0.640 & 1.916 & 0.640 & $60+$ \\
\hline Median HbA1c 9.1-10.0 \%, 76-86 (mmol/mol) & 0.974 & 0.470 & 1.951 & 0.868 & $60+$ \\
\hline Median HbA1c $>10.0 \%,>86(\mathrm{mmol} / \mathrm{mol})$ & Ref. & - & - & - & $60+$ \\
\hline Diabetes duration & 1.008 & 0.995 & 1.021 & 0.226 & $60+$ \\
\hline Male & 1.063 & 0.700 & 1.600 & 0.810 & $60+$ \\
\hline Female & Ref. & - & - & - & $60+$ \\
\hline SIMD Quintile 1 (most deprived) & Ref. & - & - & - & $60+$ \\
\hline SIMD Quintile 2 & 1.050 & 0.587 & 1.897 & 0.896 & $60+$ \\
\hline SIMD Quintile 3 & 0.561 & 0.260 & 1.154 & 0.089 & $60+$ \\
\hline SIMD Quintile 4 & 0.985 & 0.510 & 1.913 & 0.846 & $60+$ \\
\hline SIMD Quintile 5 (least deprived) & 1.012 & 0.525 & 1.929 & 0.983 & $60+$ \\
\hline Current CSII therapy & 0.702 & 0.102 & 3.003 & 0.398 & $60+$ \\
\hline Current antidepressant prescription & 2.594 & 1.714 & 3.921 & $<0.001$ & $60+$ \\
\hline Completed a structured education course & 0.761 & 0.174 & 2.483 & 0.431 & $60+$ \\
\hline
\end{tabular}

\footnotetext{
${ }^{1}$ HbA1c reference category is $>86$ due to the low number of events in the $<58$ category for certain age bands

2 CSII not included in 0-29 analysis due to low prevalence of events

${ }^{3}$ Methadone prescription not included in $60+$ analysis due to low prevalence of events
} 
ESM Table 6. Minimally adjusted association of social deprivation with each DKA outcome

\begin{tabular}{llllll}
\hline Covariate & $\exp (\mathrm{B})$ & Q2.5 & Q97.5 & Approx. p-value & Outcome \\
\hline SIMD Quintile 1 (most deprived) & Ref. & - & - & - & Any DKA Event \\
SIMD Quintile 2 & 0.703 & 0.649 & 0.762 & $<0.001$ & Any DKA Event \\
SIMD Quintile 3 & 0.525 & 0.489 & 0.563 & $<0.001$ & Any DKA Event \\
SIMD Quintile 4 & 0.430 & 0.398 & 0.463 & $<0.001$ & Any DKA Event \\
SIMD Quintile 5 (least deprived) & 0.350 & 0.314 & 0.386 & $<0.001$ & Any DKA Event \\
SIMD Quintile 1 (most deprived) & Ref. & - & - & - & DKA Case-Fatality \\
SIMD Quintile 2 & 0.836 & 0.674 & 1.036 & 0.140 & DKA Case-Fatality \\
SIMD Quintile 3 & 0.596 & 0.440 & 0.804 & $<0.001$ & DKA Case-Fatality \\
SIMD Quintile 4 & 0.853 & 0.655 & 1.120 & 0.261 & DKA Case-Fatality \\
SIMD Quintile 5 (least deprived) & 0.611 & 0.406 & 0.886 & 0.008 & DKA Case-Fatality \\
SIMD Quintile 1 (most deprived) & Ref. & - & - & - & Recurrent DKA \\
SIMD Quintile 2 & 0.583 & 0.543 & 0.625 & $<0.001$ & Recurrent DKA \\
SIMD Quintile 3 & 0.426 & 0.394 & 0.461 & $<0.001$ & Recurrent DKA \\
SIMD Quintile 4 & 0.275 & 0.248 & 0.302 & $<0.001$ & Recurrent DKA \\
SIMD Quintile 5 (least deprived) & 0.190 & 0.168 & 0.216 & $<0.001$ & Recurrent DKA \\
\hline
\end{tabular}


ESM table 7. Age-Band Stratified Recurrent DKA IRR Estimates Obtained With Multivariable Logistic Regression

\begin{tabular}{|c|c|c|c|c|c|}
\hline Covariate & IRR & Q2.5 & Q97.5 & Approx. p-value & Age Band \\
\hline Year & 1.014 & 1.005 & 1.023 & 0.003 & $0-29$ \\
\hline Age 1-10 & Ref. & - & - & - & $0-29$ \\
\hline Age 11-19 & 3.550 & 2.564 & 4.994 & $<0.001$ & $0-29$ \\
\hline Age $20-29$ & 2.438 & 1.755 & 3.457 & $<0.001$ & $0-29$ \\
\hline Median HbA1c $<7.5 \%,<58(\mathrm{mmol} / \mathrm{mol})$ & Ref. & - & - & - & $0-29$ \\
\hline Median HbA1c 7.5-9.0 \%, 58-75 (mmol/mol) & 3.018 & 2.090 & 4.469 & $<0.001$ & $0-29$ \\
\hline Median HbA1c 9.1-10.0 \%, 76-86 ( $\mathrm{mmol} / \mathrm{mol})$ & 8.739 & 6.245 & 12.703 & $<0.001$ & $0-29$ \\
\hline Median HbA1c $>10.0 \%,>86(\mathrm{mmol} / \mathrm{mol})$ & 29.522 & 21.061 & 42.769 & $<0.001$ & $0-29$ \\
\hline Diabetes duration & 0.993 & 0.986 & 1.000 & 0.041 & $0-29$ \\
\hline Male & 0.716 & 0.666 & 0.771 & $<0.001$ & $0-29$ \\
\hline Female & Ref. & - & - & - & $0-29$ \\
\hline SIMD Quintile 1 (most deprived) & Ref. & - & - & - & $0-29$ \\
\hline SIMD Quintile 2 & 0.639 & 0.576 & 0.708 & $<0.001$ & $0-29$ \\
\hline SIMD Quintile 3 & 0.532 & 0.474 & 0.597 & $<0.001$ & $0-29$ \\
\hline SIMD Quintile 4 & 0.366 & 0.319 & 0.420 & $<0.001$ & $0-29$ \\
\hline SIMD Quintile 5 (least deprived) & 0.306 & 0.257 & 0.362 & $<0.001$ & $0-29$ \\
\hline Current CSII therapy & 0.736 & 0.579 & 0.925 & 0.006 & $0-29$ \\
\hline Current methadone prescription & 2.719 & 1.629 & 4.297 & $<0.001$ & $0-29$ \\
\hline Current antidepressant prescription & 2.342 & 2.135 & 2.564 & $<0.001$ & $0-29$ \\
\hline Completed a structured education course & 0.903 & 0.754 & 1.068 & 0.221 & $0-29$ \\
\hline Year & 1.062 & 1.050 & 1.074 & $<0.001$ & $30-59$ \\
\hline Age 30-39 & Ref. & - & - & - & $30-59$ \\
\hline Age 40-49 & 0.646 & 0.576 & 0.723 & $<0.001$ & $30-59$ \\
\hline Age $50-59$ & 0.702 & 0.617 & 0.798 & $<0.001$ & $30-59$ \\
\hline Median HbA1c $<7.5 \%,<58(\mathrm{mmol} / \mathrm{mol})$ & Ref. & - & - & - & $30-59$ \\
\hline Median HbA1c 7.5-9.0 \%, 58-75 (mmol/mol) & 2.008 & 1.536 & 2.637 & $<0.001$ & $30-59$ \\
\hline Median HbA1c 9.1-10.0 \%, 76-86 ( $\mathrm{mmol} / \mathrm{mol})$ & 4.950 & 3.805 & 6.466 & $<0.001$ & $30-59$ \\
\hline Median $\mathrm{HbA} 1 \mathrm{c}>10.0 \%,>86(\mathrm{mmol} / \mathrm{mol})$ & 11.064 & 8.577 & 14.438 & $<0.001$ & $30-59$ \\
\hline Diabetes duration & 0.982 & 0.977 & 0.987 & $<0.001$ & $30-59$ \\
\hline Male & 0.755 & 0.684 & 0.833 & $<0.001$ & $30-59$ \\
\hline Female & Ref. & - & - & - & $30-59$ \\
\hline SIMD Quintile 1 (most deprived) & Ref. & - & - & - & $30-59$ \\
\hline SIMD Quintile 2 & 0.735 & 0.647 & 0.838 & $<0.001$ & $30-59$ \\
\hline SIMD Quintile 3 & 0.574 & 0.495 & 0.665 & $<0.001$ & $30-59$ \\
\hline SIMD Quintile 4 & 0.372 & 0.304 & 0.448 & $<0.001$ & $30-59$ \\
\hline SIMD Quintile 5 (least deprived) & 0.291 & 0.231 & 0.364 & $<0.001$ & $30-59$ \\
\hline Current CSII therapy & 1.245 & 0.898 & 1.687 & 0.246 & $30-59$ \\
\hline Current methadone prescription & 3.401 & 2.303 & 4.886 & $<0.001$ & $30-59$ \\
\hline Current antidepressant prescription & 2.143 & 1.925 & 2.384 & $<0.001$ & $30-59$ \\
\hline Completed a structured education course & 0.557 & 0.448 & 0.690 & $<0.001$ & $30-59$ \\
\hline Year & 1.092 & 1.064 & 1.120 & $<0.001$ & $60+$ \\
\hline Age 60-69 & Ref. & - & - & - & $60+$ \\
\hline Age $70-79$ & 1.512 & 1.208 & 1.892 & 0.001 & $60+$ \\
\hline Age $80+$ & 2.068 & 1.542 & 2.734 & $<0.001$ & $60+$ \\
\hline Median HbA1c $<7.5 \%,<58(\mathrm{mmol} / \mathrm{mol})$ & Ref. & - & - & - & $60+$ \\
\hline Median HbA1c 7.5-9.0 \%, 58-75 (mmol/mol) & 2.271 & 1.527 & 3.506 & $<0.001$ & $60+$ \\
\hline Median HbA1c 9.1-10.0 \%, 76-86 $(\mathrm{mmol} / \mathrm{mol})$ & 5.131 & 3.386 & 8.040 & $<0.001$ & $60+$ \\
\hline Median HbA1c $>10.0 \%,>86(\mathrm{mmol} / \mathrm{mol})$ & 9.630 & 6.452 & 14.913 & $<0.001$ & $60+$ \\
\hline Diabetes duration & 1.011 & 1.004 & 1.018 & 0.002 & $60+$ \\
\hline Male & 0.774 & 0.628 & 0.950 & 0.017 & $60+$ \\
\hline Female & Ref. & - & - & - & $60+$ \\
\hline SIMD Quintile 1 (most deprived) & Ref. & - & - & - & $60+$ \\
\hline SIMD Quintile 2 & 0.979 & 0.707 & 1.352 & 0.927 & $60+$ \\
\hline SIMD Quintile 3 & 0.776 & 0.562 & 1.065 & 0.103 & $60+$ \\
\hline SIMD Quintile 4 & 0.878 & 0.636 & 1.216 & 0.406 & $60+$ \\
\hline SIMD Quintile 5 (least deprived) & 0.660 & 0.457 & 0.943 & 0.016 & $60+$ \\
\hline Current CSII therapy & 1.074 & 0.375 & 2.524 & 0.765 & $60+$ \\
\hline Current antidepressant prescription & 2.171 & 1.707 & 2.749 & $<0.001$ & $60+$ \\
\hline Completed a structured education course & 0.368 & 0.167 & 0.712 & 0.003 & $60+$ \\
\hline
\end{tabular}

${ }^{1}$ Methadone prescription not included in $60+$ analysis due to low prevalence of events 
ESM table 8. DKA Event (excluding events at time of diagnosis) IRR Estimates Obtained With Multivariable Poisson Regression

\begin{tabular}{lllll}
\hline Covariate & IRR & Q2.5 & Q97.5 & Approx. p-value \\
\hline Year & 1.050 & 1.046 & 1.054 & $<0.001$ \\
Age 1-10 & Ref. & - & - & - \\
Age 11-19 & 1.950 & 1.729 & 2.201 & $<0.001$ \\
Age 20-29 & 1.257 & 1.110 & 1.424 & $<0.001$ \\
Age 30-39 & 0.751 & 0.658 & 0.857 & $<0.001$ \\
Age 40-49 & 0.603 & 0.526 & 0.692 & $<0.001$ \\
Age 50-59 & 0.552 & 0.479 & 0.641 & $<0.001$ \\
Age 60-69 & 0.558 & 0.478 & 0.651 & $<0.001$ \\
Age 70-79 & 0.826 & 0.698 & 0.978 & 0.036 \\
Age 80+ & 1.381 & 1.129 & 1.686 & 0.002 \\
Median HbA1c $<7.5 \%,<58(\mathrm{mmol} / \mathrm{mol})$ & Ref. & - & - & - \\
Median HbA1c 7.5-9.0 \%, 58-75 (mmol/mol) & 1.725 & 1.609 & 1.848 & $<0.001$ \\
Median HbA1c 9.1-10.0\%, 76-86 (mmol/mol) & 3.138 & 2.918 & 3.375 & $<0.001$ \\
Median HbA1c >10.0 \%, >86 (mmol/mol) & 5.419 & 5.025 & 5.832 & $<0.001$ \\
Diabetes duration & 0.996 & 0.994 & 0.999 & 0.004 \\
Male & 0.803 & 0.763 & 0.846 & $<0.001$ \\
Female & Ref. & - & - & - \\
SIMD Quintile 1 (most deprived) & Ref. & - & - & - \\
SIMD Quintile 2 & 0.741 & 0.688 & 0.802 & $<0.001$ \\
SIMD Quintile 3 & 0.572 & 0.533 & 0.613 & $<0.001$ \\
SIMD Quintile 4 & 0.481 & 0.445 & 0.518 & $<0.001$ \\
SIMD Quintile 5 (least deprived) & 0.414 & 0.375 & 0.456 & $<0.001$ \\
Current CSII therapy & 0.915 & 0.842 & 0.994 & 0.033 \\
Current methadone prescription & 1.449 & 1.219 & 1.715 & $<0.001$ \\
Current antidepressant prescription & 1.476 & 1.429 & 1.524 & $<0.001$ \\
Completed a structured education course & 0.890 & 0.831 & 0.951 & 0.001 \\
\hline
\end{tabular}


ESM table 9. Case-Fatality (with DKA as underlying cause) Odds-Ratio Estimates Obtained With Multivariable Logistic Regression

\begin{tabular}{lllll}
\hline Covariate & OR & Q2.5 & Q97.5 & Approx. p-value \\
\hline Year & 1.030 & 1.005 & 1.055 & 0.017 \\
Age & 1.043 & 1.036 & 1.050 & $<0.001$ \\
Median HbA1c $<7.5 \%,<58(\mathrm{mmol} / \mathrm{mol})$ & Ref. & - & - & - \\
Median HbA1c $7.5-9.0 \%, 58-75(\mathrm{mmol} / \mathrm{mol})$ & 0.676 & 0.443 & 1.076 & 0.129 \\
Median HbA1c $9.1-10.0 \%, 76-86(\mathrm{mmol} / \mathrm{mol})$ & 0.703 & 0.448 & 1.165 & 0.224 \\
Median HbA1c $>10.0 \%,>86(\mathrm{mmol} / \mathrm{mol})$ & 0.807 & 0.541 & 1.272 & 0.420 \\
Diabetes duration & 1.008 & 0.999 & 1.016 & 0.073 \\
Male & 2.313 & 1.877 & 2.860 & $<0.001$ \\
Female & Ref. & - & - & - \\
SIMD Quintile 1 (most deprived) & Ref. & - & - & - \\
SIMD Quintile 2 & 0.883 & 0.681 & 1.139 & 0.377 \\
SIMD Quintile 3 & 0.587 & 0.406 & 0.829 & 0.003 \\
SIMD Quintile 4 & 0.885 & 0.634 & 1.218 & 0.399 \\
SIMD Quintile 5 (least deprived) & 0.704 & 0.469 & 1.040 & 0.079 \\
Current CSII therapy & 0.598 & 0.236 & 1.307 & 0.153 \\
Current methadone prescription & 5.196 & 2.913 & 8.730 & $<0.001$ \\
Current antidepressant prescription & 2.001 & 1.625 & 2.459 & $<0.001$ \\
Completed a structured education course & 0.856 & 0.488 & 1.421 & 0.432 \\
\hline
\end{tabular}

\section{ESM Figure Captions}

- ESM Figure 1 - Distribution of DKA risk factors over the study period, calculated within the population of people with type 1 diabetes residing in each quintile of social deprivation. a) Ever completed a structured education course, b) Prescription for an antidepressant in a given year, c) Prescription for methadone in a given year, d) use of CSII in a given year.

- ESM Figure 2 - Directly standardised diabetic ketoacidosis (DKA) event rate over the study period, separated by sex and also calculated for both males and females jointly. Vertical lines represent $95 \%$ confidence intervals for the standardised rate. The rate of DKA events for females always exceeded that of males, and all rates experienced an upward trend over the study period.

- ESM Figure 3 - The percentage of diagnoses of type 1 diabetes that occurred with a concurrent diabetic ketoacidosis (DKA) hospital admission. The solid line represents the modelled trend over the study period, and the shaded area represents the $95 \%$ credibility interval $(95 \% \mathrm{CrI})$ for this trend. The modelled trend suggests a well-defined increase in the rate at which individuals are diagnosed with type 1 diabetes during a concurrent DKA hospital admission.

- ESM Figure 4 - Directly standardised diabetic ketoacidosis (DKA) mortality rate over the study period, separated in to 10 year age bands.

\section{References}

1. Bürkner P-C. Brms : An R Package for Bayesian Multilevel Models Using Stan. Journal of Statistical Software [Internet]. 2017 [cited 2021 May 10];80(1). Available from: http://www.jstatsoft.org/v80/i01/

2. Honaker J, King G, Blackwell M. Amelia II: A Program for Missing Data. Journal of Statistical Software, Articles [Internet]. 2011;45(7):1-47. Available from: https://www.jstatsoft.org/v045/i07 